\title{
On Harmonic Index and Diameter of Quasi-Tree Graphs
}

\author{
A. Abdolghafourian and Mohammad A. Iranmanesh $(D)$ \\ Department of Mathematics, Yazd University, 89195-741 Yazd, Iran \\ Correspondence should be addressed to Mohammad A. Iranmanesh; iranmanesh@yazd.ac.ir
}

Received 23 December 2020; Revised 23 January 2021; Accepted 31 January 2021; Published 16 February 2021

Academic Editor: Ismail Naci Cangul

Copyright (C 2021 A. Abdolghafourian and Mohammad A. Iranmanesh. This is an open access article distributed under the Creative Commons Attribution License, which permits unrestricted use, distribution, and reproduction in any medium, provided the original work is properly cited.

The harmonic index of a graph $G(H(G))$ is defined as the sum of the weights $2 /\left(d_{u}+d_{v}\right)$ for all edges $u v$ of $G$, where $d_{u}$ is the degree of a vertex $u$ in $G$. In this paper, we show that $H(G) \geq D(G)+5 / 3-(n / 2)$ and $H(G) \geq((1 / 2)+(2 / 3(n-2))) D(G)$, where $G$ is a quasi-tree graph of order $n$ and diameter $D(G)$. Indeed, we show that both lower bounds are tight and identify all quasi-tree graphs reaching these two lower bounds.

\section{Introduction}

Let $G$ be a simple connected graph with vertex set $V(G)$ and edge set $E(G)$ of order $n(|V(G)|=n)$. The harmonic index of $G$, first appeared in [1], is defined as $H(G)=\sum_{u v \in E(G)} 2 /\left(d_{u}+d_{v}\right)$, where for $v \in V(G), d_{v}$ is the degree of $v$ in $G$. For $u, v \in V(G)$, the distance between $u$ and $v$ is shown by $d(u, v)$. Also, $D(G)=\max \{d(u, v)\}_{u, v \in V(G)}$ is the diameter of $G$ and $\delta(G)=\min \left\{d_{v}\right\}_{v \in V(G)}$.

The applications of the harmonic index in various chemical disciplines have been demonstrated in [2-4]. Also, several studies have focused on graph theoretical properties of the harmonic index, see, for example, [5-9]. For a broad overview, we refer to [10].

A connected graph $G$ is a quasi-tree graph if $G$ is not a tree and there exists a vertex $v \in V(G)$ such that $G-v$ is a tree. A graph $G$ is called unicyclic if it contains only one cycle. Obviously, every unicyclic graph is a quasi-tree graph. Many researchers have studied topological indices of quasitree graphs. See, for example, [11-16].

Liu [17] found a relation between harmonic index and diameter of a graph. He proved that if $n \geq 4$ and $G$ is a connected graph of order $n$, then $H(G) \leq D(G)+(n / 2)-1$ and $H(G) \leq(n / 2) D(G)$. Also, a lower bound was found for trees. If $T$ is a tree of order $n \geq 4$, then $H(T) \geq D(T)+(5 / 6)-(n / 2) \quad$ and $H(T) \geq((1 / 2)$ $+(1 / 3(n-1))) D(T)$. Thereby, Liu [17] proposed the following conjecture.

Conjecture 1. Let $G$ be a connected graph with order $n \geq 4$; then,

$$
\begin{aligned}
& H(G) \geq D(G)+\frac{5}{6}-\frac{n}{2}, \\
& H(G) \geq\left(\frac{1}{2}+\frac{1}{3(n-1)}\right) D(G) .
\end{aligned}
$$

Jerline and Michaelraj $[18,19]$ found a sharper bound for unicyclic graphs. They showed that if $G$ is a unicyclic graph of order $n$, then $H(G) \geq D(G)+5 / 3-(n / 2)$ and $H(G) \geq((1 / 2)+(2 / 3(n-2))) D(G)$. They introduced a family of graphs, $U_{n, 4}^{1, n-5}$, which is a set of graphs obtained from $C_{4}$ by attaching one pendant vertex and a path of length $n-5$ to two diametrically nonadjacent vertices of $C_{4}$ 



Figure 1: The graphs $U_{n, 4}^{1, n-5}$ and $V_{r, s}$.

(see Figure 1). Then, they proposed the following conjecture [18].

Conjecture 2. Let $G$ be a simple connected graph, which is not a tree, of order $n \geq 7$. Then,

$$
\begin{aligned}
& H(G) \geq D(G)+\frac{5}{3}-\frac{n}{2}, \\
& H(G) \geq\left(\frac{1}{2}+\frac{2}{3(n-2)}\right) D(G),
\end{aligned}
$$

where equality holds if and only if $G=U_{n, 4}^{1, n-5}$.

They also show that the inequalities of the above conjecture are not true for a graph of order 6 , namely, $U_{6,4}^{1,1}$.

Suppose $K_{4}^{-}$is a graph of order 4 which is obtained from $K_{4}$ by deleting an edge. Also, for $r, s \geq 0$, let $V_{r, s}$ be a family of graphs obtained from $K_{4}^{-}$by attaching two paths of lengths $r$ and $s$ to two nonadjacent vertices of $K_{4}^{-}$(see Figure 1 ). We will show that the inequality holds for all quasi-tree graphs except $U_{6,4}^{1,1}$ and the graph $U_{5,3}^{1,1}$ which is obtained by attaching two pendant vertices to two vertices of $K_{3}$. Also, the equality holds for $V_{1,1}$.
Two main theorems of this paper are as follows.

Theorem 1. Let $G \neq U_{6,4}^{1,1}, U_{5,3}^{1,1}$ be a quasi-tree graph of order $n \geq 3$. Then, $H(G) \geq D(G)+5 / 3-(n / 2)$. The equality holds if and only if $G=V_{1,1}$ or $U_{n, 4}^{1, n-5}$.

Theorem 2. Let $G$ be a quasi-tree graph of order $n \geq 3$ and $G \neq U_{6,4}^{1,1}, U_{5,3}^{1,1}$. Then, $H(G) \geq((1 / 2)+(2 / 3(n-2))) D(G)$. The equality holds if and only if $G=V_{1,1}$ or $U_{n, 4}^{1, n-5}$.

In Section 2, we prove the lemmas that will be used in Section 3, where we prove the main theorems.

All graphs considered in this paper are finite, undirected, connected, and simple. Let $G$ be a graph and $v \in V(G)$ and $P$ a path of $G$; then, by $G-v$ and $G-P$, we mean the graph obtained from $G$ by deleting the vertex $v$ and the vertices of $P$, respectively. For all other notation and definitions not given here, the readers are referred to [20].

\section{Preliminaries}

Lemma 1. For $x, y \geq 2$, the two variables' function

$$
f(x, y)=\frac{x+4}{x(x+1)(2+x)}+\frac{y+4}{y(y+1)(2+y)}-\frac{2}{(x+y)(x+y-2)}
$$

is positive.

Proof

$$
\begin{aligned}
f(x, y)= & \frac{x+4}{x(x+1)(2+x)}+\frac{y+4}{y(y+1)(2+y)}-\frac{2}{(x+y)(x+y-2)} \\
= & \frac{2}{x(x+1)}+\frac{-1}{(x+2)(x+1)}+\frac{2}{y(y+1)}+\frac{-1}{(y+2)(y+1)}-\frac{2}{(x+y)(x+y-2)} \\
= & \left(\frac{1}{x(x+1)}-\frac{1}{(x+2)(x+1)}\right)+\left(\frac{1}{y(y+1)}-\frac{1}{(y+2)(y+1)}\right) \\
& +\left(\frac{1}{x(x+1)}-\frac{1}{(x+y)(x+y-2)}\right)+\left(\frac{1}{y(y+1)}-\frac{1}{(x+y)(x+y-2)}\right) .
\end{aligned}
$$


Given $x, y \geq 2$, the terms inside parentheses are positive.

Lemma 2. $x /(x+2) \geq 1 /(5+x)+(x-1) /(2+x) \geq 11 / 28$ for every $x \geq 2$.

Proof. The first inequality is valid since $x /(x+2)$ $=1 /(x+2)+(x-1) /(x+2) \geq 1 /(5+x)+(x-1) /(2+x)$.

Let $\quad f(x)=1 /(5+x)+(x-1) /(2+x)$. Then, $f^{\prime}(x)=\left(-1 /(5+x)^{2}\right)+\left(3 /(2+x)^{2}\right)>0$. So, $f$ is an increasing function and $f(x) \geq f(2)=(11 / 28)$ for every $x \geq 2$.

\section{Proof of the Main Theorems}

In this section, we will show that Conjecture 2 is true for all quasi-tree graphs. Also, in our proof, it will be shown that the equality in both inequalities hold whenever $G$ is the graph $V_{1,1}$.

Lemma 3. Let $G$ be a quasi-tree graph of order $n$, where $3 \leq n \leq 6$, such that $G \neq U_{6,4}^{1,1}, U_{5,3}^{1,1}$. Then,

$$
\begin{aligned}
& H(G) \geq D(G)+\frac{5}{3}-\frac{n}{2}, \\
& H(G) \geq\left(\frac{1}{2}+\frac{2}{3(n-2)}\right) D(G) .
\end{aligned}
$$

Proof. If $n=3$, then $G$ should be the complete graph, $K_{3}$. In this case, $D(G)=1$ and, by an easy calculation, $H(G)=$ $(3 / 2)$ and both inequalities hold.

If $n=4$, then, since $K_{4}$ is not a quasi-tree graph, $D(G)>1$. Also, since $G$ is not a tree, $G \neq P_{4}$ and $D(G)=2$. Hence, $\quad D(G)+5 / 3-(n / 2)=((1 / 2)+(2 / 3(n-2))) D(G)$ $=5 / 3$. So, both inequalities hold for $n=4$ (see Figure 2 ).

Suppose $n=5$ and $w$ is the vertex that $G-w$ is a tree. Since $G-w$ is a tree of order $4, D(G-w)=3$ or 2 . Also, since $G$ is a quasi-tree graph, it is not a complete graph, and hence, $D(G)=3$ or 2 .

If $D(G)=3$, then $D(G)+5 / 3-(n / 2)=((1 /$ $2)+(2 / 3(n-2))) D(G)=13 / 6$. If $D(G)=2$, then $D(G)+$ $5 / 3-(n / 2)=(7 / 6)$ and $((1 / 2)+(2 / 3(n-2))) D(G)=$ (13/9). All quasi-tree graphs of order 5 and their harmonic indices are shown in Figure 3. As it is seen, all graphs hold both inequalities except when $G=U_{5,3}^{1,1}$.

Suppose $n=6$ and $w$ is the vertex that $G-w$ is a tree. So, $G-w$ is one of $P_{5}, K_{1,4}$, or $K_{1,3}^{+}$, where $K_{1,3}^{+}$is obtained by attaching a new pendant vertex to a pendant vertex of $K_{1,3}$.

If $\quad G-w=K_{1,4}$, then $D(G-w)=2$. Since $D(G) \leq D(G-w)$ and $G$ is not $K_{6}$, so $D(G)=2$, $D(G)+5 / 3-(n / 2)=(2 / 3), \quad$ and $\quad((1 / 2)+(2 / 3(n-2)))$ $D(G)=(4 / 3)$. On the contrary, for every edge $u v$ of $G-w$, $\left(2 / d_{u}+d_{v}\right)$ is at least $(2 / 7)$, where $d_{u}$ and $d_{v}$ are the degree of $u$ and $v$ in $G$, respectively. So,

$$
H(G) \geq 4\left(\frac{2}{7}\right)+\sum_{x \in N(w)} \frac{2}{d_{x}+d_{w}} \geq \frac{8}{7}+\frac{2}{5+d_{w}}+\frac{2\left(d_{w}-1\right)}{2+d_{w}} \geq \frac{8}{7}+\frac{11}{14}>\frac{4}{3}
$$

The second and third inequalities hold by Lemma 2 .

If $G-w=P_{5}$, then the graph $G$ is one of the graphs shown in Figure 4 in which their harmonic indices are calculated. Also, $D(G) \leq 4$, so $D(G)+5 / 3-(n / 2)$ $\leq 2+(2 / 3)$ and $((1 / 2)+(2 / 3(n-2))) D(G) \leq 2+(2 / 3)$. So, for every graph both inequalities hold, except when $G=U_{6,4}^{1,1}$. Also, the equality holds when $G=V_{1,1}$.

If $G-w=K_{1,3}^{+}$, then the graph $G$ is one of the graphs shown in Figure 5 in which their harmonic indices are calculated. Also, $D(G) \leq 3$, so $D(G)+5 / 3-(n / 2) \leq$ $1+(2 / 3)$ and $((1 / 2)+(2 / 3(n-2))) D(G) \leq 2$. Obviously, for every graph, both inequalities hold.
Theorem 3. Let $G \neq U_{6,4}^{1,1}, U_{5,3}^{1,1}$ be a quasi-tree graph with $n \geq 3$ vertices. Then, $H(G) \geq D(G)+5 / 3-(n / 2)$. The equality holds if and only if $G=V_{1,1}$ or $G=U_{n, 4}^{1, n-5}$.

Proof. By induction on $n$, if $n \leq 6$, then Theorem 3 implies that the inequality is true, unless when $G=U_{6,4}^{1,1}, U_{5,3}^{1,1}$. Also, by Theorem 3, the equality holds when $G=V_{1,1}$.

Let $G$ be a quasi-tree graph with $n \geq 7$ vertices. Suppose $w$ is a vertex of $G$ such that $G-w$ is a tree. Let $P=u_{0}-u_{1}-$ $\cdots-u_{d}$ be the diametrical path of $G$. There are three cases as follows. 

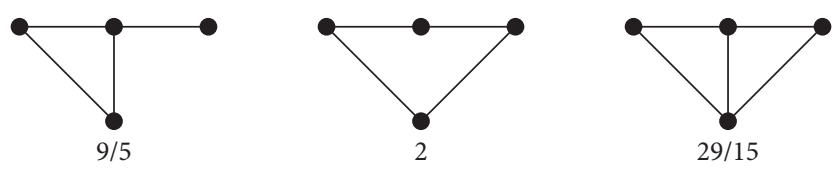

Figure 2: Quasi-tree graphs of order 4 and their harmonic indices.
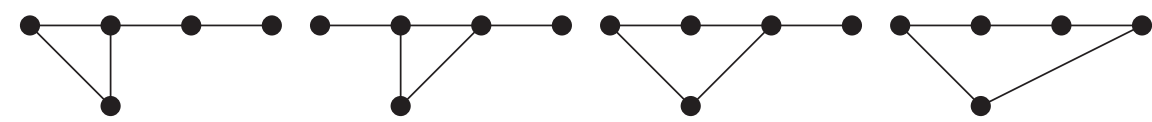

$71 / 30$

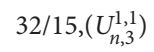

$23 / 10$

$5 / 2$
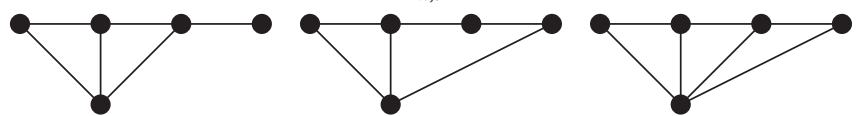

$83 / 35$

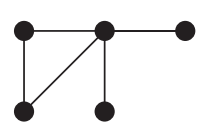

$23 / 10$

$73 / 30$

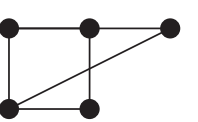

$59 / 30$

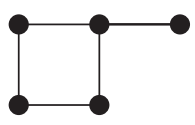

$23 / 10$

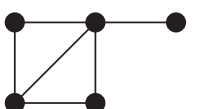

$226 / 105$

$12 / 5$

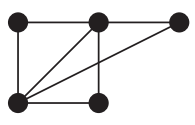

$9 / 4$

FIGURE 3: Quasi-tree graphs of order 5 and their harmonic indices.
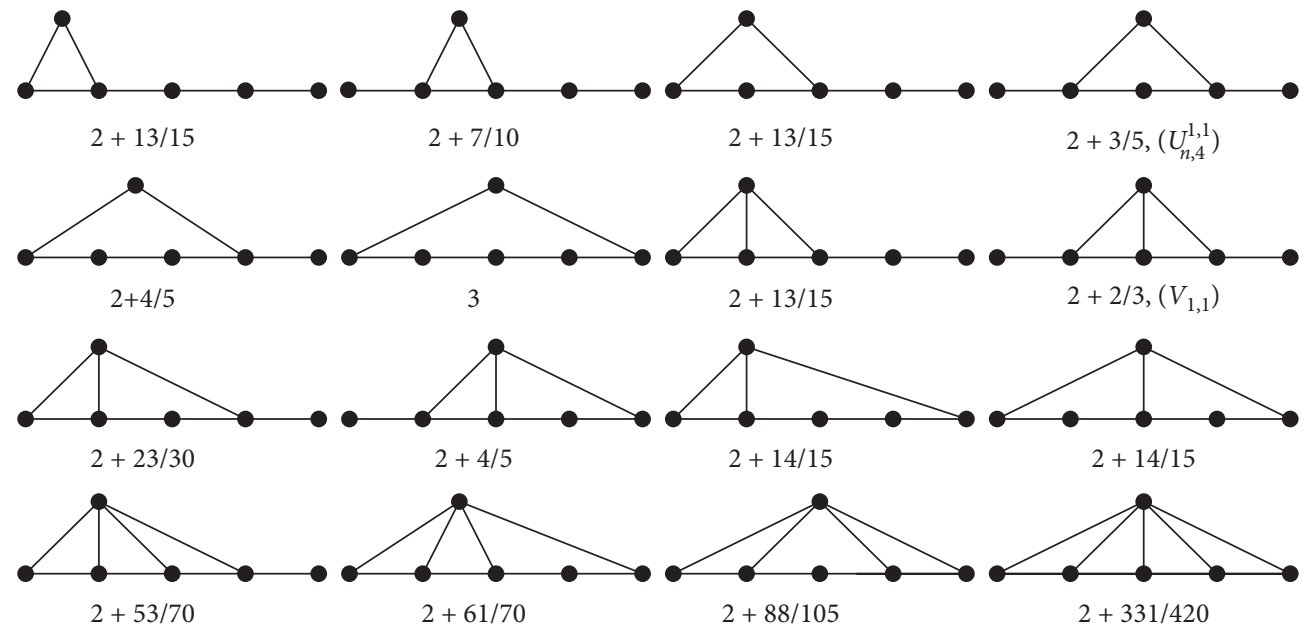

Figure 4: Quasi-tree graphs of order 6 obtained from $P_{5}$, and their harmonic indices.
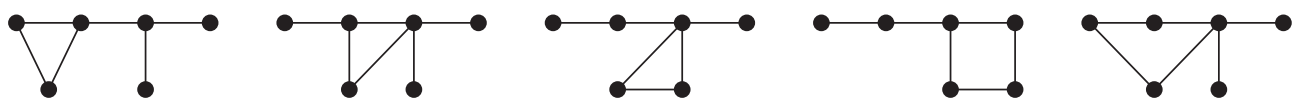

$2+19 / 30$
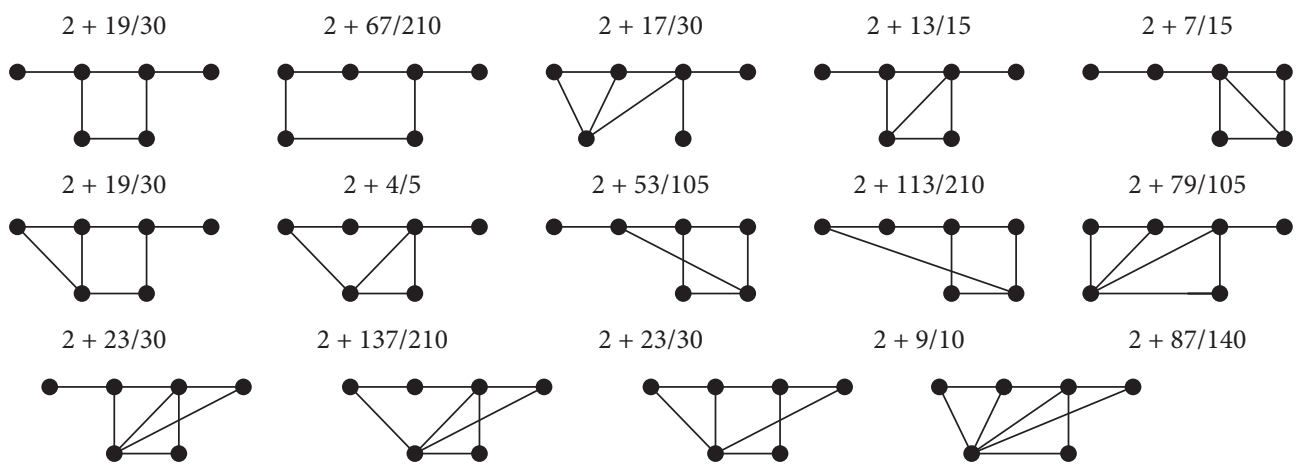

$2+137 / 210$

$2+23 / 30$

$2+9 / 10$
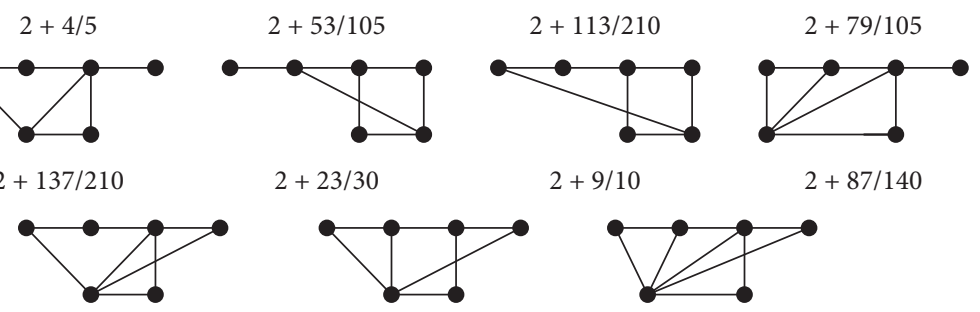

$2+86 / 105$

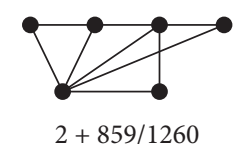

FIGURE 5: Quasi-tree graphs of order 6 obtained from $K_{1,3}^{*}$, and their harmonic indices. 
Case 1. There exists $t \in G-P$ such that $d_{t}=1$. Since $t$ is not in diametrical path of $G$ and $d_{t}=1, D(G-t)=$ $D(G)$ and $G-t$ is a quasi-tree graph too. Suppose
$N(t)=\{r\}$ and $G-t \neq U_{6,4}^{1,1}$. Then, by induction hypothesis,

$$
\begin{aligned}
H(G)= & H(G-t)+\frac{2}{d_{r}+1}-\sum_{\substack{x \in N(r) \\
x \neq t}} \frac{2}{d_{r}+d_{x}-1}+\sum_{\substack{x \in N(r) \\
x \neq t}} \frac{2}{d_{r}+d_{x}} \\
= & H(G-t)+\frac{2}{d_{r}+1}-\sum_{x \in N(r)} \frac{2}{\left(d_{r}+d_{x}-1\right)\left(d_{r}+d_{x}\right)} \geq H(G-t) \\
& +\frac{2}{d_{r}+1}-\frac{2\left(d_{r}-1\right)}{d_{r}\left(d_{r}+1\right)} \geq\left(D(G)+\frac{5}{3}-\frac{n-1}{2}\right)+\frac{2}{d_{r}\left(d_{r}+1\right)}>D(G)+\frac{5}{3}-\frac{n}{2} .
\end{aligned}
$$

If $G-t=U 6,4^{1,1}$, then $G$ is one of the graphs shown with their harmonic indices in Figure 6. In this case, $D(G)=4$ and $D(G)+5 / 3-(n / 2)=(65 / 30)$. Hence, the inequality holds.

Case 2. Every vertex of $G-P$ is of degree at least 2, and there exists $t \in G-P-w$ such that $d_{t}=2$. Similar to the previous case, $D(G)=D(G-t)$. Suppose $N(t)=$ $\{r, s\}$. Note that $d_{r}, d_{s} \geq 0$, otherwise $\{r, s\} \cap P=\varnothing$, and hence, $t \in P$, a contradiction. Since $d_{t}=2$, it is possible that $G-t$ be a tree. There exist three subcases as follows:

Subcase 2.1. G- $t$ is not a tree and $r$ and $s$ are not adjacent in $G$. By the hypothesis, there exist at most two vertices of degree 1 in $G$ which are $u_{0}$ and $u_{d}$. If $u_{0}, u_{d} \in N(r)$, then $D(G)=2$, and since $r$ and $s$ are not adjacent, $d\left(s, u_{0}\right) \geq 3$, a contradiction. So, $r$ has at most one neighbor of degree 1 . Same argument is valid for $s$, so

$$
\begin{aligned}
H(G)= & H(G-t)+\frac{2}{2+d_{r}}+\frac{2}{2+d_{s}}-2 \sum_{\substack{x \in N(r) \\
x \neq t}} \frac{1}{\left(d_{r}-1+d_{x}\right)\left(d_{r}+d_{x}\right)} \\
& -2 \sum_{y \in N(s)} \frac{1}{\left(d_{s}-1+d_{y}\right)\left(d_{y}+d_{s}\right)} \geq D(G)+\frac{5}{3}+\frac{n-1}{2}+\frac{2}{2+d_{r}}+\frac{2}{2+d_{s}} \\
& -\frac{2\left(d_{r}-2\right)}{\left(d_{r}+1\right)\left(d_{r}+2\right)}-\frac{2}{d_{r}\left(d_{r}+1\right)}-\frac{2\left(d_{s}-2\right)}{\left(d_{s}+1\right)\left(d_{s}+2\right)}-\frac{2}{d_{s}\left(d_{s}+1\right)} \\
= & D(G)+\frac{5}{3}-\frac{n}{2}+\frac{1}{2}+\frac{4\left(d_{r}-1\right)}{d_{r}\left(d_{r}+1\right)\left(2+d_{r}\right)}+\frac{4\left(d_{s}-1\right)}{d_{s}\left(d_{s}+1\right)\left(2+d_{s}\right)}>D(G)+\frac{5}{3}-\frac{n}{2}
\end{aligned}
$$




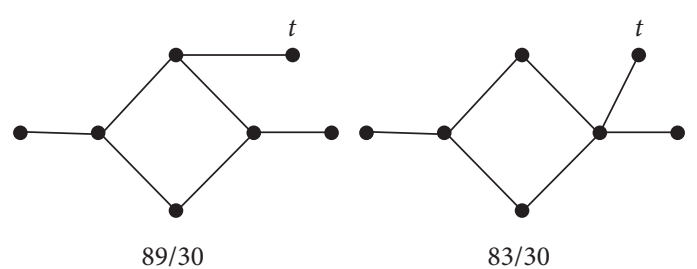

Figure 6: The graphs related to Case 1 of Theorems 3 and 4 .

If $G-t=U_{6,4}^{1,1}$, then $G$ is one of the graphs which are shown with their harmonic indices in Figure 7 . In this case, $D(G)=4$ and $D(G)+5 / 3-(n / 2)=(65 / 30)$.
Subcase 2.2. $G-t$ is not a tree and $r$ and $s$ are adjacent in $G$. If $G-t \neq U_{6,4}^{1,1}$, then

$$
\begin{aligned}
H(G)= & H(G-t)+\frac{2}{d_{r}+2}+\frac{2}{d_{s}+2}+\frac{2}{d_{r}+d_{s}}-\frac{2}{d_{r}+d_{s}-2} \\
& -\sum_{x \in N(r)} \frac{2}{\left(d_{x}+d_{r}-1\right)\left(d_{x}+d_{r}\right)}-\sum_{\substack{y \in N(s) \\
y \neq t, r}} \frac{2}{\left(d_{y}+d_{s}-1\right)\left(d_{y}+d_{s}\right)} \\
\geq & H(G-t)+\frac{2}{d_{r}+2}+\frac{2}{d_{s}+2}-\frac{4}{\left(d_{r}+d_{s}\right)\left(d_{r}+d_{s}-2\right)}-\frac{2\left(d_{r}-2\right)}{d_{r}\left(d_{r}+1\right)}-\frac{2\left(d_{s}-2\right)}{d_{s}\left(d_{s}+1\right)} \\
\geq & H(G-t)-\frac{2\left(d_{r}+4\right)}{\left(d_{r}+d_{s}\right)\left(d_{r}+d_{s}-2\right)}+\frac{2\left(d_{s}+4\right)}{d_{r}\left(d_{r}+1\right)\left(d_{r}+2\right)}+\frac{2}{d_{s}\left(d_{s}+1\right)\left(d_{s}+2\right)} \\
> & D(G)+\frac{5}{3}-\frac{n}{2} .
\end{aligned}
$$

Since $d_{r}, d_{s} \geq 2$, the last inequality is obtained from Lemma 1.

If $G-t=U_{6,4}^{1,1}$, then $G$ is the graph which is shown with its harmonic index in Figure 8. In Subcase 2.1, $D(G)+5 / 3-(n / 2)=(65 / 30)$ and the inequality holds.

Subcase 2.3. $G-t$ is a tree. Since $G-w$ is also a tree, by counting the number of edges and vertices, it is obtained that $d_{w}=d_{t}=2$. This means that $G$ is a unicyclic graph and as proved by Theorem 3 in[19], $H(G) \geq D(G)+5 / 3-(n / 2)$, in which equality holds if $G=U_{n, 4}^{1, n-5}$

Case 3. Every vertex of $G-\left\{u_{0}, u_{1}, \ldots, u_{d}\right\}$ is of degree at least 2 and if $t \in V(G)$ and $d_{t}=2$, then $t \in\left\{u_{0}, u_{1}, \ldots, u_{d}, w\right\}$. Since $G-w$ is a tree, every pendant vertex of $G-w$ is in $\left\{u_{0}, u_{1}, \ldots, u_{d}\right\}$. So, $G-w$ is a path and $V(G)=\left\{u_{0}, u_{1}, \ldots, u_{d}\right\} \cup\{w\}$. Also, since $G$ is not a tree $d_{w} \geq 2$. If $d_{w}=2$, then $G$ is a unicyclic graph and as proposed by Theorem 3 in [19], $H(G) \geq D(G)+5 / 3-(n / 2)$, with equality holds if $G=U_{n, 4}^{1, n-5}$. So, there exist two subcases as follows.

Subcase $3.1 d_{w}=3$. In this case, $G$ is one of the graphs in Table 1 . In all cases, $H(G) \geq D(G)+5 / 3-(n / 2)$. As it is shown, the equality holds when $G=V_{1,1}$.
Subcase $3.2\left(d_{w}>3\right)$. Suppose $\left\{u_{h}, u_{i}, u_{j}, u_{k}\right\} \subseteq N(w)$ such that $h<i<j<k$. If $k-h>2$, then the diametrical path $u_{1}-\cdots-u_{h}-\cdots-u_{k}-\cdots-u_{d}$ is longer than the path $u_{1}-\cdots-u_{h}-w-u_{k}-\cdots, u_{d}$, a contradiction. So, $k-h \leq 2$, which is another contradiction. Hence, this case does not happen. The inequality holds in all cases and equality holds if and only if $G=V_{1,1}$ or $U_{n, 4}^{1, n-5}$.

Theorem 4. Let $G \neq U_{6,4}^{1,1}, U_{5,3}^{1,1}$ be a quasi-tree graph with $n \geq 3$ vertices, then

$$
H(G) \geq\left(\frac{1}{2}+\frac{2}{3(n-2)}\right) D(G) .
$$

The equality holds if and only if $G=V_{1,1}$ or $U_{n, 4}^{1, n-5}$.

Proof. The proof is similar to the proof of Theorem 3. By induction on $n$, if $n \leq 6$, then Theorem 3 implies that the inequality holds unless when $G=U_{6,4}^{1,1}, U_{5,3}^{1,1}$.

Let $G$ be a quasi-tree graph with $n \geq 7$ vertices. Suppose $w$ is a vertex of $G$ such that $G-w$ is a tree. Let $P=u_{0}-u_{1}-$ $\cdots-u_{d(G)}$ be the diametrical path of $G$. Since $G-w$ is a tree, $\delta(G) \leq 2$. There exist three cases as follows. 


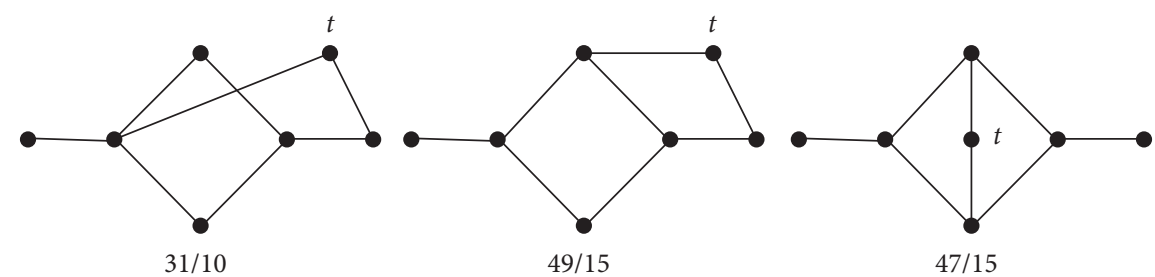

Figure 7: The graphs related to Subcase 2.1 of Theorems 3 and 4.

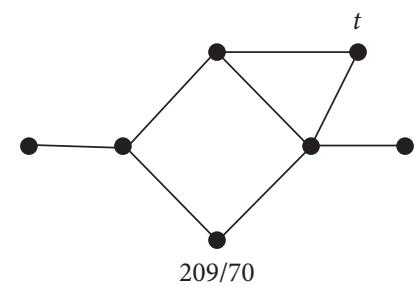

Figure 8: The graphs related to Subcase 2.2 of Theorems 3 and 4.

Table 1: All possibilities for Case 3 of Theorems 3 and 4.

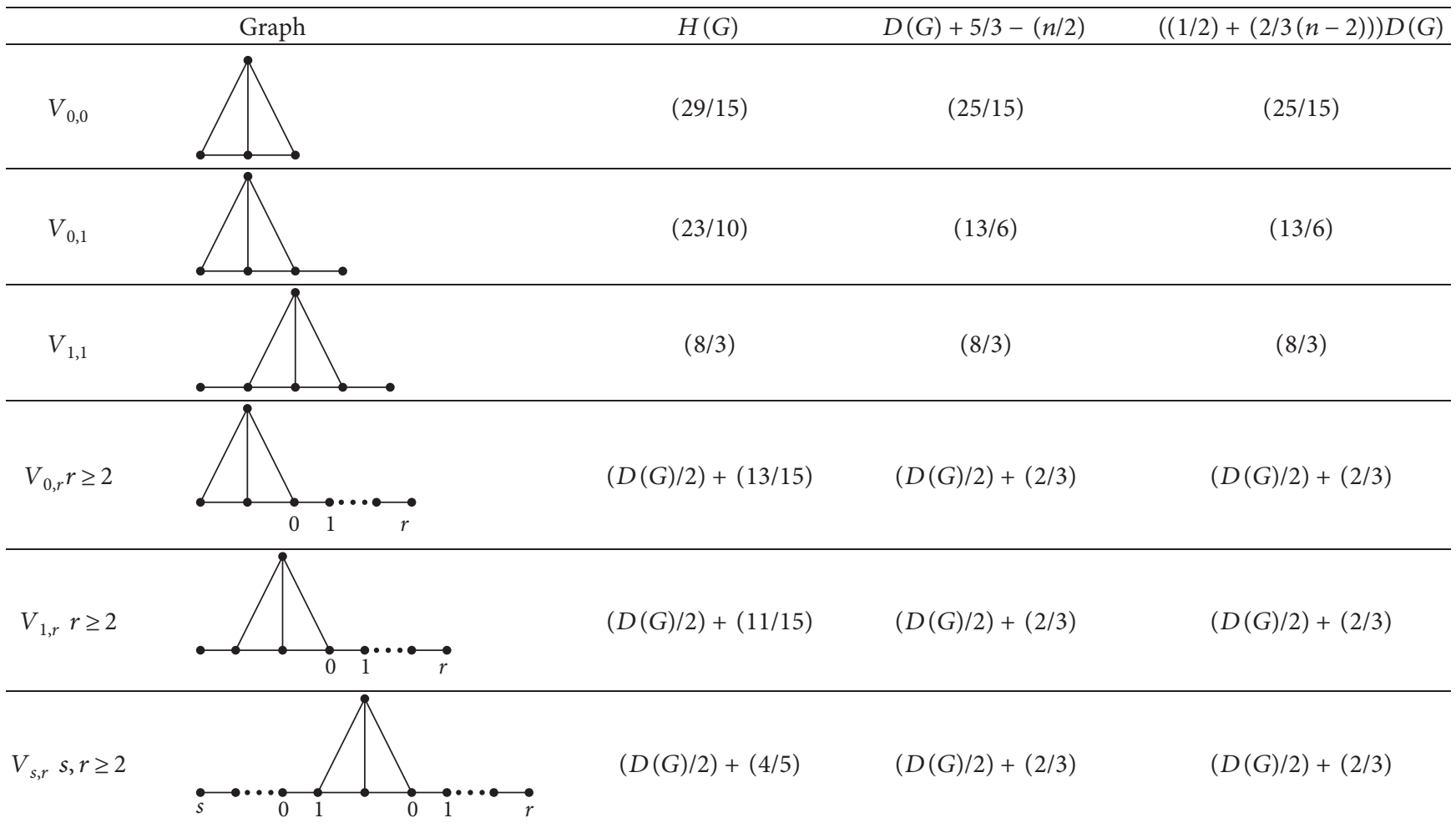

Case 1. There exists $t \in G-P$ such that $d_{t}=1$. Since $t$ is not in diametrical path of $G, D(G)=D(G-t)$. Suppose $N(t)=r$. So, such as Case 1 in the proof of
Theorem 3, if $G-t \neq U_{6,4}^{1,1}$, then, by induction hypothesis,

$$
H(G) \geq H(G-t)+\frac{2}{d_{r}\left(d_{r}+1\right)}>\left(\frac{1}{2}+\frac{2}{3(n-3)}\right) D(G)>\left(\frac{1}{2}+\frac{2}{3(n-2)}\right) D(G)
$$


If $G-t=U_{6,4}^{1,1}$, then $G$ is one of the graphs which is shown with their harmonic indices in Figure 6. In this case, $D(G)=4$ and $((1 / 2)+(2 / 3(n-2))) D(G)=$ $(38 / 15)$. Hence, the inequality holds.

Case 2. Every vertex of $G-P$ is of degree at least 2, and there exists a vertex $t \in G-P-w$ such that $d_{t}=2$. From the previous case, $D(G)=D(G-t)$. Suppose
$N(t)=\{r, s\}$. Since $d_{t}=2$, it is possible that $G-t$ be a tree. There exist three subcases as follows.

Subcase 2.1. $G-t$ is not a tree and $r$ and $s$ are not adjacent in G. By the same argument as in Subcase 2.1 of Theorem 3,

$$
H(G)>H(G-t)+\frac{4\left(d_{r}-1\right)}{d_{r}\left(d_{r}+1\right)\left(2+d_{r}\right)}+\frac{4\left(d_{s}-1\right)}{d_{s}\left(d_{s}+1\right)\left(2+d_{s}\right)}>\left(\frac{1}{2}+\frac{2}{3(n-2)}\right) D(G) .
$$

If $G-t=U_{6,4}^{1,1}$, then $((1 / 2)+(2 / 3(n-2))) D(G)=$ $(38 / 15)$, and $G$ is one of the graphs which are shown with their harmonic indices in Figure 7.
Subcase 2.2. $G-t$ is not a tree and $r$ and $s$ are adjacent in G. By Subcase 2.2 of Theorem 3,

$$
H(G) \geq H(G-t)+\frac{2\left(d_{r}+4\right)}{d_{r}\left(d_{r}+1\right)\left(d_{r}+2\right)}+\frac{2\left(d_{s}+4\right)}{d_{s}\left(d_{s}+1\right)\left(d_{s}+2\right)}-\frac{4}{\left(d_{r}+d_{s}\right)\left(d_{s}+d_{r}-2\right)}>H(G-t)
$$

So, by the induction hypothesis,

$H(G)>\left(\frac{1}{2}+\frac{2}{3(n-3)}\right) D(G)>\left(\frac{1}{2}+\frac{2}{3(n-2)}\right) D(G)$.

If $G-t=U_{6,4}^{1,1}$, then $((1 / 2)+(2 / 3(n-2))) D(G)=$ $(38 / 15)$ and $G$ is the graph which is shown with its harmonic index in Figure 8 and the inequality holds. Subcase 2.3. $G-t$ is a tree. By the same argument as in the proof of Subcase 2.3 of Theorem 3, G is a unicyclic graph and by Theorem 3 in [18], $H(G) \geq$ $((1 / 2)+(2 / 3(n-2))) D(G)$, with equality when $G=U_{n, 4}^{1, n-5}$

Case 3. Every vertex of $G-P$ is of degree at least 2, and if $t \in V(G)$ and $d_{t}=2$, then $t \in\left\{u_{0}, u_{1}, \ldots, u_{d}, w\right\}$. By the same argument as in Case 3 of Theorem $3, G-w$ is a path and $V(G)=t \in\left\{u_{0}, u_{1}, \ldots, u_{d}\right\} \cup\{w\}$. Also, since $G$ is not a tree, $d_{w} \geq 2$. If $d_{w}=2$, then $G$ is a unicyclic graph and by Theorem 3 in[18], $H(G) \geq((1 / 2)+$ $(2 / 3(n-2))) D(G)$, with equality if $G=V_{1,1}$ or $G=U_{n, 4}^{1, n-5}$. Also, by Subcase 3.2 of Theorem $3, d_{w} \ngtr 3$. So, $d_{w}=3$ and $G$ is one of the graphs in Table 1 . Obviously, $H(G) \geq((1 / 2)+(2 / 3(n-2))) D(G)$, for all of them. As in Theorem 3, the inequality holds in all cases and the equality will be satisfy if and only if $G=$ $V_{1,1}$ or $U_{n, 4}^{1, n-5}$.

\section{Data Availability}

No data were used to support this study.

\section{Disclosure}

An earlier version of this manuscript has been presented as arXiv in Semantic Scholar according to this link.

\section{Conflicts of Interest}

The authors declare that they have no conflicts of interest.

\section{References}

[1] S. Fajtlowicz, "On conjectures of Graffiti-II," Congr Numer, vol. 60, pp. 187-197, 1987.

[2] C. Betancur, R. Cruz, and J. Rada, "Vertex-degree-based topological indices over starlike trees," Discrete Applied Mathematics, vol. 185, pp. 18-25, 2015.

[3] B. Furtula, I. Gutman, and M. Dehmer, "On structure-sensitivity of degree-based topological indices," Applied Mathematics and Computation, vol. 219, no. 17, pp. 8973-8978, 2013.

[4] J. Rada and R. Cruz, "Vertex-degree-based topological indices over graphs," MATCH Commun. Math. Comput. Chem.vol. 72, no. 3, pp. 603-616, 2014.

[5] A. Ali, S. Balachandran, S. Elumalai, and T. Mansour, "On the n-vertex trees with sixth to fifteenth maximum harmonic indices," Afrika Matematika, vol. 31, pp. 771-780, 2019.

[6] H. Deng, S. Balachandran, S. K. Ayyaswamy, and Y. B. Venkatakrishnan, "On the harmonic index and the chromatic number of a graph," Discrete Applied Mathematics, vol. 161, no. 16-17, pp. 2740-2744, 2013.

[7] H. Deng, T. Vetrik, and S. Balachandran, "On the harmonic index and diameter of unicyclic graphs," Mathematical Reports, vol. 22, no. 72, pp. 11-18, 2020.

[8] J.-B. Lv and J. Li, "The harmonic index of a graph and its DP-chromatic number," Discrete Applied Mathematics, vol. 284, pp. 611-615, 2020.

[9] I. R. Abdolhosseinzadeh, F. Rahbarnia, and M. Tavakoli, "Some vertex-degree-based topological indices under edge corona product," Italian Journal of Pure and Applied Mathematics, vol. 38, pp. 81-91, 2017.

[10] A. Ali, L. Zhong, and I. Gutman, "Harmonic index and its generalizations: extremal results and bounds," MATCH Communications in Mathematical and in Computer Chemistry, vol. 81, no. 2, pp. 249-311, 2019. 
[11] M. A. Iranmanesh and R. Nejati, "On the Estrada index of point attaching strict k-quasi tree graphs," Kragujevac Journal of Mathematics, vol. 44, no. 2, pp. 165-179, 2020.

[12] S. Li, X. Li, and W. Jing, "On the extremal MerrifieldSimmons index and Hosoya index of quasi-tree graphs," Discrete Applied Mathematics, vol. 157, no. 13, pp. 28772885, 2009.

[13] S. N. Qiao, "On the Zagreb index of quasi-tree graphs," Applied Mathematics E-Notes, vol. 10, pp. 147-150, 2010.

[14] S. N. Qiao, "On zeroth-order general Randić index of quasitree graphs containing cycles," Discrete Optimization, vol. 7, no. 3, pp. 93-98, 2010.

[15] S. Wagner and I. Gutman, "Maxima and minima of the Hosoya index and the Merrifield-Simmons index," Acta Applicandae Mathematicae, vol. 112, no. 3, pp. 323-346, 2010.

[16] K. Xu, H. Liu, and K. C. Das, "The Kirchhoff index of quasi-tree graphs," Zeitschrift für Naturforschung A, vol. 70, no. 3, pp. 135-139, 2015.

[17] J. Liu, "On harmonic index and diameter of graphs," Journal of Applied Mathematics and Physics, vol. 1, no. 3, pp. 5-6, 2013.

[18] J. A. Jerline and L. B. Michaelraj, "On harmonic index and diameter of unicyclic graphs," Iranian Journal of Mathematical Sciences and Informatics, vol. 11, no. 1, pp. 115-122, 2016.

[19] J. A. Jerline and L. B. Michaelraj, "On a conjecture of harmonic index and diameter of graphs," Kragujevac Journal of Mathematics, vol. 40, no. 1, pp. 73-78, 2016.

[20] D. B. West, Introduction To Graph Theory, Vol. 2, PrenticeHall, Upper Saddle River, USA, 2001. 